\title{
通
}

\section{Perspectivas y desafíos para la competitividad de las Mipymes de la cadena del cuero de Chocontá (Cundinamarca)}

Perspectives and challenges for competitiveness of MSMES Chain Leather Chocontá (Cundinamarca)

Ludwin Alexander Sánchez Vargas*

* Administrador de Empresas, Especialista en Finanzas, Magister en Administración MBA. Docente de la Universidad Nacional Abierta y a Distancia - UNAD. Correo electrónico: ludwin.sanchez@unad.edu.co. 


\section{Introducción}

Las perspectivas y los desafíos para la competitividad de las Mipymes curtidoras de Chocontá (Cundinamarca, Colombia), planteadas en el presente documento, se derivan del proyecto de investigación"Fortalecimiento de la gestión empresarial de Mipymes de la cadena del cuero del municipio de Chocontá (Cundinamarca)", desarrollado por el grupo de investigación Fénix de la Escuela de Ciencias Administrativas, Contables, Económicas y de Negocios - ECACEN de la Universidad Nacional Abierta y a Distancia. Este análisis contiene la descripción del programa de acompañamiento para el fortalecimiento de la gestión empresarial, el mejoramiento de las prácticas productivas, logísticas y de comercialización para promover el uso de tecnologías limpias, propiciar el acceso a mercados nacionales e internacionales y contribuir a mejorar la competitividad de esas Mipymes.

Como contribución al desarrollo de ese municipio, a la provincia de Almeidas (ubicada en el departamento de Cundinamarca) y a la región cundiboyacense de Colombia, se proponen lineamientos para la permanencia y el crecimiento de las organizaciones estudiadas. Con esto además se generará una transformación productiva que propicie el crecimiento económico y el desarrollo humano.

Este texto presenta el Programa de Acompañamiento para el Fortalecimiento a la Gestión Empresarial de Mipymes Curtidoras del Municipio de Chocontá (Cundinamarca), a partir de los hallazgos encontrados en la caracterización realizada a 13 Mipymes curtidoras de cuero de ese municipio. Este proceso permitió visualizar las perspectivas y los desafíos que tienen esas empresas y diseñar el programa de asesoría que, se espera, contribuya al mejoramiento de una gestión que se anticipe al futuro.

Para contextualizar al lector acerca de la investigación realizada, a continuación se muestran algunos indicadores de la cadena productiva del cuero en Colombia. Este sector aporta el 0,27\% del PIB nacional, el 2,17\% al PIB manufacturero y el o,6\% del empleo nacional (Propaís y Raddar Ltda., 2015). No obstante, de acuerdo con el DANE, "la producción de cuero en Colombia registró un decrecimiento de $-15,6 \%$ entre enero y abril de 2017. Respecto a 
las ventas se observa una variación de -24,3\%. El empleo cae en un 4,4\%" (Citado en Asociación Colombiana de Industriales del Calzado, el Cuero y sus Manufacturas - ACICAM, 2017, p. 3). Asimismo, según ACICAM.

Las exportaciones de cuero en enero a abril de 2017 ascienden a 36.5 millones de dólares con una variación de $-20,7 \%$ respecto al mismo periodo de 2016. De estas US $\$ 8.3$ millones corresponden a cuero crudo y US\$17,2 millones a wet blue.

El principal país destino de las exportaciones de cuero es China con una participación del $41 \%$ y un crecimiento de $83 \%$, seguido de Italia $20 \%$ y Hong Kong $6 \%$ que registran variaciones de $-33 \%$ y $-54 \%$, respectivamente; entre otros destinos.

El mayor departamento exportador de Cuero es Atlántico (43\%), le sigue en orden de importancia Bogotá y Cundinamarca (23\%) y Antioquia (22\%). Las importaciones de cuero en enero a abril de 2017 ascienden a 1,9 millones de dólares con una disminución de $-40 \%$, frente a los 3,1 millones de dólares registrados en el mismo lapso de 2016.

El principal país de origen es Uruguay (24\%), le siguen Italia (16\%), Colombia (por procesos de reimportación 12\%), Estados Unidos y Puerto Rico (10\%) y Brasil (9\%) (2017, p. 2).

Como es lógico, este panorama plantea desafíos para la competitividad. Máxime si se revisa con detenimiento la tabla 1, en la que se consolida la caracterización de las 13 empresas curtidoras de cuero, que hacen parte de las 36 ubicadas en la vereda Chingacío de Chocontá (Cundinamarca) y que pertenecen a la Asociación Ecológica de Chingacío - ASECHI. Empresas cuyas principales fortalezas son: a) conocimientos y buena actitud del talento humano, b) preparación de la fuerza comercial, c) infraestructura física, d) conocimiento de las necesidades en términos operativos y administrativos y e) experiencia en el sector. No obstante, las debilidades más sobresalientes son: a) procesos de producción no óptimos; b) falta de capacitación en gestión gerencial, técnica (procesos y productos) y operativa; c) escases de capital de trabajo (en especial para financiar ventas a crédito); d) incapacidad para cumplir las normas ambientales; e) endeudamiento con entidades financieras y $f$ ) escases de recursos para inversión. 
También enfrentan las siguientes oportunidades y amenazas. Las oportunidades más relevantes son: a) crecimiento de las exportaciones gracias a la globalización, b) cumplimiento del sello verde y c) mercado internacional abierto y en crecimiento. Sin embargo, las amenazas más importantes son: a) exportación condicionada a la operación legal, b) cierre por la aplicación estricta de las normas ambientales, c) competencia desleal, d) informalidad empresarial, e) acaparamiento de pieles por grandes empresas y capitalistas que generan escases y altos precios, f) competencia agresiva de mayoristas y empresas exportadoras de Ibagué (Colombia), g) devaluación, h) precios de los insumos, i) exportación del cuero en pelo, j) importación de artículos de cuero (calzado de China), k) contrabando de productos de cuero que ingresan al país y l) solicitud de crédito (financiación) por parte de los clientes, que limita la competencia con empresas que sí lo ofrecen.

Tabla 1. Caracterización de las empresas curtidoras de cuero de Chocontá

\begin{tabular}{|c|c|c|c|}
\hline Característica & $\frac{\frac{1}{\pi}}{\frac{10}{2}}$ & Característica & 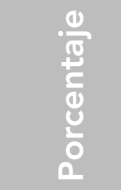 \\
\hline Antigüedad & $\begin{array}{l}15 \text { a } 35 \text { años: } \\
46,15 \% \\
\text { No informa: } \\
38,46 \% \\
\text { En } 2013: 7,69 \% \\
\text { Hace } 5 \text { años: } \\
7,69 \%\end{array}$ & $\begin{array}{l}\text { Claridad en la estructura } \\
\text { de costos }\end{array}$ & $84,62 \%$ \\
\hline No. de empleados & $\begin{array}{l}\text { De } 1 \text { a } 20: 69,23 \% \\
\text { a destajo } \\
\text { Uno: } 7,69 \% \\
25: 7,69 \%\end{array}$ & $\begin{array}{l}\text { Elaboración presupuestos de } \\
\text { ingresos y egresos }\end{array}$ & $30,77 \%$ \\
\hline $\begin{array}{l}\text { Contratación formal de } \\
\text { empleados }\end{array}$ & $15,38 \%$ & $\begin{array}{l}\text { Aplicación de principios de } \\
\text { contabilidad generalmente } \\
\text { aceptada }\end{array}$ & $30,77 \%$ \\
\hline Tipo de empresa & $\begin{array}{l}\text { Micro: } 92,31 \% \\
\text { Pequeña: } 7,69 \%\end{array}$ & $\begin{array}{l}\text { Puntualidad en pago de } \\
\text { impuestos y obligaciones legales }\end{array}$ & $69,23 \%$ \\
\hline $\begin{array}{l}\text { Criterios técnicos de empaque } \\
\text { del cuero }\end{array}$ & $7,69 \%$ & $\begin{array}{l}\text { Sistema formal de control } \\
\text { a la gestión }\end{array}$ & $7,69 \%$ \\
\hline
\end{tabular}




\begin{tabular}{|c|c|c|c|}
\hline $\begin{array}{l}\text { Formulación formal y } \\
\text { conocimiento de misión, visión, } \\
\text { valores, principios, objetivos, } \\
\text { por el grupo social }\end{array}$ & $7,69 \%$ & $\begin{array}{l}\text { Balance General y Estados de } \\
\text { Resultados }\end{array}$ & $7,69 \%$ \\
\hline Políticas de gestión & $7,69 \%$ & Cálculo de indicadores financieros & $15,38 \%$ \\
\hline $\begin{array}{l}\text { Medición de resultados } \\
\text { (obtenidos vs. propuestos) }\end{array}$ & $15,38 \%$ & $\begin{array}{l}\text { Análisis de resultados financieros } \\
\text { y comparación con presupuestos }\end{array}$ & $23,08 \%$ \\
\hline $\begin{array}{l}\text { Conocimiento de fortalezas y } \\
\text { debilidades }\end{array}$ & $53,85 \%$ & Portafolio de productos & $0 \%$ \\
\hline $\begin{array}{l}\text { Conocimiento de } \\
\text { oportunidades y amenazas }\end{array}$ & $69,23 \%$ & $\begin{array}{l}\text { Manuales de procesos y } \\
\text { procedimientos }\end{array}$ & $23,08 \%$ \\
\hline $\begin{array}{l}\text { Asignación de recursos para } \\
\text { mercadeo y publicidad, }\end{array}$ & $0 \%$ & $\begin{array}{l}\text { Sistemas de información } \\
\text { confiables y oportunos para } \\
\text { tomar decisiones }\end{array}$ & $15,38 \%$ \\
\hline Metas de ventas & $0 \%$ & $\begin{array}{l}\text { Planes de control para } \\
\text { optimización de recursos }\end{array}$ & $23,08 \%$ \\
\hline Políticas de servicio al cliente & $0 \%$ & $\begin{array}{l}\text { Certificación Normas Técnicas de } \\
\text { Calidad (Icontec, ISO, OSHAS, } \\
\text { etc.) }\end{array}$ & $0 \%$ \\
\hline $\begin{array}{l}\text { Existencia estructura comercial } \\
\text { para el mercadeo }\end{array}$ & $0 \%$ & Capacitación a su personal & $7,69 \%$ \\
\hline Plan de mercadeo & $7,69 \%$ & Evaluación a proveedores & $15,38 \%$ \\
\hline $\begin{array}{l}\text { Conocimiento Cadena del } \\
\text { Cuero }\end{array}$ & $\begin{array}{l}\text { Con claridad: } \\
38,46 \% \\
\text { En parte: } 15,38 \% \\
\text { No: } 46,15 \%\end{array}$ & $\begin{array}{l}\text { Licencias, títulos, permisos, } \\
\text { autorizaciones y/o concesiones } \\
\text { para uso y aprovechamiento de } \\
\text { recursos naturales y del medio } \\
\text { ambiente }\end{array}$ & $30,77 \%$ \\
\hline $\begin{array}{l}\text { Conocimiento necesidades de } \\
\text { los clientes }\end{array}$ & No: $7,69 \%$ & $\begin{array}{l}\text { Permisos de vertimientos, } \\
\text { tratamiento de aguas residuales y } \\
\text { sostenibilidad del recurso hídrico }\end{array}$ & $7,69 \%$ \\
\hline $\begin{array}{l}\text { Conocimiento de los } \\
\text { competidores }\end{array}$ & $84,62 \%$ & $\begin{array}{l}\text { Definición estructura } \\
\text { organizacional, cargos y perfiles }\end{array}$ & $0 \%$ \\
\hline $\begin{array}{l}\text { Propuesta de valor clara para } \\
\text { los clientes }\end{array}$ & $46,15 \%$ & $\begin{array}{l}\text { Cumplimiento Código Sustantivo } \\
\text { de Trabajo }\end{array}$ & $7,69 \%$ \\
\hline $\begin{array}{l}\text { Conocimiento posicionamiento } \\
\text { y participación en el mercado }\end{array}$ & $30,77 \%$ & $\begin{array}{l}\text { Programas de capacitación para } \\
\text { el personal }\end{array}$ & $0 \%$ \\
\hline Evaluación del desempeño & $7,69 \%$ & $\begin{array}{l}\text { Programas de bienestar para los } \\
\text { empleados y su núcleo familiar }\end{array}$ & $0 \%$ \\
\hline Planes de incentivos & $0 \%$ & $\begin{array}{l}\text { Percepción de un ambiente de } \\
\text { trabajo adecuado }\end{array}$ & $30,77 \%$ \\
\hline
\end{tabular}

Fuente: elaboración propia 
Como se observa, existen factores sociales, económicos, financieros, políticos, legales, ambientales y culturales que plantean riesgos de permanencia y crecimiento. Aunque existen fortalezas, hay debilidades mayores que limitan mucho el desempeño y que se deben atender de forma inmediata. De esta forma, perspectivas como exportar y obtener el sello verde son escenarios lejanos; principalmente por la incapacidad para cumplir las normas ambientales en cuanto a licencias, títulos, permisos, autorizaciones o concesiones para uso y aprovechamiento de los recursos naturales y del medio ambiente, tratamiento de aguas residuales, vertimientos, sostenibilidad del recurso hídrico y sistemas de producción limpia. Circunstancias que, sumadas a la fluctuación de la disponibilidad de materia prima directa y al trabajo ilegal que se realiza en horas nocturnas para evadir los controles de la autoridad ambiental, ponen en entredicho la continuidad de estas empresas y la producción de cueros curtidos.

Ninguna de las empresas caracterizadas desarrolla procesos formales de planeación, y mucho menos planeación estratégica. De modo que no se cuenta con una visión de un futuro deseado, que sea probable y posible de alcanzar. Tampoco existen procesos de gestión que identifiquen claramente funciones como planeación, organización, dirección y control. Los procedimientos contables y financieros son muy precarios y no son utilizados para tomar decisiones ni para optimizar el control y los procesos comerciales que permitan alcanzar la satisfacción de los clientes.

En atención a que el objetivo general de la investigación es fortalecer la gestión empresarial, el mejoramiento de la productividad y la competitividad de las Mipymes curtidoras de cuero de Chocontá, en el proceso administrativo (planeación, organización, dirección y control) y en las áreas funcionales (producción, mercadeo y ventas, contabilidad y finanzas, gestión del talento humano), los objetivos específicos propuestos son los siguientes: diagnosticar las empresas; diseñar el programa de fortalecimiento; acompañar la formulación y la implementación de planes estratégicos y tácticos; y medir el avance de cada empresa frente al objetivo del programa. De modo que, en cumplimiento del segundo objetivo específico, el autor presenta el Programa 
de Acompañamiento para el Fortalecimiento a la Gestión Empresarial de Mipymes Curtidoras del Municipio de Chocontá.

\section{Marco teórico}

Como fundamento teórico para desarrollar la investigación y diseñar el Programa deAcompañamiento para el Fortalecimiento a la Gestión Empresarial de Mipymes Curtidoras del Municipio de Chocontá (Cundinamarca), se tuvieron en cuenta el pensamiento estratégico, la dirección estratégica y la gerencia basada en el valor. En los siguientes acápites se hace un esbozo de cada uno de estos temas.

\section{Pensamiento estratégico}

Si el pensamiento común y corriente se entiende como el de la cotidianidad, que es natural, predecible, repetitivo y automático para una misma situación y como un proceso deliberado, dinámico y continuo; el pensamiento estratégico se asume como la forma de concebir un contexto determinado. Llevado al mundo de las organizaciones y las empresas, el pensamiento estratégico es la perspectiva que tienen los directivos de organizaciones y empresas para analizar los aspectos cualitativos del negocio y su entorno para definir la visión de futuro a la que esperan Ilegar. Como lo plantea Román, "en el mundo de los negocios el pensamiento estratégico se asocia con el proceso intelectual intuitivo, divergente, imaginativo, análogo y simultáneo, el cual busca combinar los métodos analíticos con la elasticidad mental" (2010, p. 28).

La planeación estratégica debe concebirse como medio para formular estrategias y propiciar la coordinación de todo el grupo social de la organización hacia la superación de la competencia como objetivo. Así que, en aspectos como el conocimiento del negocio, se involucra un conocimiento que, según Minstzberg, Quinn y Voyer, "no es conocimiento intelectual, no son reportes analíticos o hechos y cifras abstractas (aunque éstas ciertamente pueden ayudar); se trata de un conocimiento personal, de una comprensión íntima, 
equivalente a la sensibilidad del alfarero hacia el barro. Los hechos son accesibles a cualquiera; no así este tipo de conocimiento: sabiduría es la palabra que mejor lo describe" (1997, p. 129).

En palabras de Román, formular estrategias es un proceso compuesto por"una serie de decisiones que sigan la siguiente lógica: visión estratégica, misión o finalidad de la organización, objetivos, ámbito de la empresa, posicionamiento competitivo, metas estratégicas y estrategias y planes de acción de respaldo" (2010, p. 26). Entonces, el pensamiento estratégico exige que la labor gerencial se encamine hacia la generación de valor, no solamente para accionistas, sino para clientes, empleados y todos los grupos de interés, pues demanda un "enfoque sistémico, capacidad de síntesis, inteligencia intuitiva y creatividad con el fin de visualizar un futuro y articular la forma de llegar a él" (p. 26).

Según Robert (2006), el pensamiento estratégico es un proceso que exige el estudio de la organización y su entorno para reunir toda la información disponible, ponerla en perspectiva, separar la información pertinente de la inadecuada y obtener conclusiones racionales para formular la estrategia. Además, de acuerdo con Robert (2006), es un ejercicio de sentido común y prosperidad, de fácil entendimiento, que conjuga conocimiento y experiencia de los administradores y de todo el grupo social. De modo que permite tomar opiniones e información subjetiva que - acompañada de conocimientos, experiencia yjuicios objetivosse convierte en insumo para la toma de decisiones racionales sobre el futuro.

Por obvias razones, es importante tener en cuenta que el pensamiento estratégico debe irradiar desde la alta dirección, siendo este el nivel responsable del diseño de la estrategia de la organización. Este pensamiento exige la identificación precisa de productos, clientes, segmentos de mercado, mercados geográficos y, en general, el entorno para la formulación de estrategias. Por consiguiente, en palabras de Román (2010), "quienes desempeñan la función gerencial deben disponer de un tipo de pensamiento muy particular para definir las estrategias que sus empresas requieren para ser exitosas en este medio tan competitivo" (p. 24). 
De manera que, desde la concepción de Robert (2006), el pensamiento estratégico permite que la formulación de la estrategia sea participativa y entendida por todo el grupo social para su implementación. Participación que debe ser motivada y facilitada por los directivos para que la visión de la organización sea un objetivo común e inspire su actuación. Esto también hará que las empresas curtidoras de Chocontá puedan llevar a cabo lo que plantea Román, en el sentido de pensar estratégicamente, pues

La clave del éxito de una empresa no radica de forma exclusiva en lo económico y en el saber producir bien, sino también en saber vender bien tanto el producto como su propia imagen al traslucir su responsabilidad social, y esto se logra mediante la generación de calidad de vida, progreso y desarrollo para los empleados, clientes, proveedores y demás grupos de interés que interactúan en la organización (2011, p. 49).

Por consiguiente, los empresarios y directivos deben ser proactivos y asumir el pensamiento estratégico como medio para entender la realidad y propender por el futuro deseado.

\section{Dirección estratégica}

Este estilo de gestión se concibe como responsabilidad del más alto nivel, por parte de cualquier organización o empresa cuya tarea sea la de formular y ejecutar estrategias para el logro de la visión perseguida. Es decir, en palabras de Dess y Lumpkin, aborda "objetivos globales de la organización, incluye múltiples grupos de interés en la empresa, incorpora perspectivas tanto a corto como a largo plazo, y reconoce la necesidad de compensaciones entre la eficacia y la eficiencia" (2003, p. 3). De manera que la gerencia se integre con las áreas funcionales (producción mercadotecnia, contabilidad y finanzas, talento humano, etc.) para "que la organización pueda funcionar como una máquina bien aceitada" (Laudon y Laudon, 2004, p. 51).

Así se pueden crear y conservar ventajas competitivas que, según Hill y McShane (2008), constituyen la preponderancia de una empresa al superar a 
sus rivales; propósito que deben asumir las empresas curtidoras de Chocontá, pues se deben poner en marcha tácticas competitivas o "acciones, de corto plazo o largo plazo, orientadas a superar los rivales y que pueden hacerse con base en la fijación de precios y la oferta de productos" (Hill y McShane, 2008, p. 147). Por tanto, la dirección estratégica debe entenderse "como el conjunto de análisis, decisiones y acciones que una organización lleva a cabo para crear y mantener ventajas competitivas" (Dess y Lumpkin, 2003, p. 3) y lograr la visión de la organización. Proceso que requiere de una descripción precisa del concepto de negocio y de la toma de decisiones óptimas, tanto en épocas favorables como en aquellas de dificultades (Robert, 2006). Además, tal como lo proponen Dess y Lumpkin, "la dirección estratégica de una organización supone tres procedimientos continuos: análisis, decisión y acción" (2003, p. 8)

Para evitar riesgos es fundamental medir y prever las implicaciones de las estrategias que serán formuladas y ejecutadas, que, en términos generales, pueden clasificarse como estrategias FO, FA, DO y DA. Las FO sugieren emplear las fortalezas para aprovechar las oportunidades. Las FA apuntan a minimizar las amenazas a partir de las fortalezas. Las DO se orientan a superar las debilidades para aprovechar las oportunidades. Y las DA exigen extinguir las debilidades para combatir las amenazas.

En este contexto es necesario precisar que la dirección estratégica es más que la mera planeación estratégica. Según Chiavenato y Sapiro, la planeación estratégica exige una gran reflexión "acerca de la intención estratégica de la organización" (2011, p. 73), que "también incluye una orientación general hacia el crecimiento" (Harrison y St. John, 2002, p. 61). Entonces, la planeación estratégica debe entenderse como "el arte y la ciencia de formular, implementar y evaluar decisiones multidisciplinarias que permiten que una empresa alcance sus objetivos" (David, 2013, p. 5).

No obstante, es importante tener en cuenta que la planeación estratégica marca la pauta de la dirección estratégica porque en ella se realiza el diagnóstico de la organización y se formulan las estrategias a ejecutar. Para esto es crucial el diagnóstico externo, en el que se desarrollan tres grandes pasos: 
a) Resumen y evaluación externa indirecta, a través de "información económica, social, cultural, demográfica, ambiental, política, gubernamental, legal, tecnológica y competitiva" (David, 2013, p. 80); b) identificación de las cinco fuerzas competitivas de Porter (1998), a saber: competidores actuales, competidores potenciales, bienes sustitutos, proveedores y clientes, a través de las que se "determina la rentabilidad de la industria" (Flores, Salazar y Tapia, 2011, p. 29); y c) evaluación externa directa por medio de los factores claves de éxito (FCE) que determinan lo que las empresas deben hacer de forma efectiva para generar valor y que, según Romero, Noriega, Escobar y Ávila (2009), "aportan información valiosa para alcanzar las metas y objetivos de la empresa" (p. 7). Igualmente, se debe efectuar una evaluación interna que "sintetiza y evalúa las fortalezas y debilidades más importantes encontradas en las áreas funcionales de una empresa y también constituye la base para identificar y evaluar las relaciones entre estas áreas" (David, 2013, p. 122).

\section{Gerencia basada en el valor}

Entendida como la gerencia basada en la creación de valor, se puede asumir como la que permite obtener "riqueza, teniendo en cuenta la eficiencia y la productividad de sus activos, así como la estructura de capital y el entorno dentro del cual se mueve" (Ortiz, 2003, p. 215). De manera que, en términos de Gutiérrez (2003), se busque la supervivencia y el crecimiento. Objetivo hacia el que deben orientarse las empresas curtidoras de Chocontá.

No obstante, la gestión basada en el valor debe concebirse como proceso continuo, lógico y sistemático que reduzca la incertidumbre al máximo posible y propicie el logro de los objetivos organizacionales. De forma tal que se satisfagan las necesidades y expectativas de los grupos de interés (clientes externos, clientes internos, inversionistas, etc.). Es decir, "crear riqueza, teniendo en cuenta la eficiencia y la productividad de sus activos, así como la estructura de capital y el entorno dentro del cual se mueve" (Ortiz, 2003, p. 215). En palabras de Hill y McShane (2008), superar a los rivales a través del desarrollo y la conservación de ventajas competitivas. 
Es fundamental que cada unidad curtidora de cuero tenga clara su cadena de valor, la cual, en esencia, está constituida por actividades primarias y actividades de apoyo. Las primarias "tienen que ver con el diseño, la creación y la entrega del producto; su comercialización; y su apoyo y servicio post-venta" (Hill y McShane, 2008, p. 144); estas además involucran Investigación y Desarrollo $(I+D)$, producción, marketing, ventas y servicio al cliente. Las segundas, es decir las de apoyo, son abastecimiento, logística, sistemas de información y recursos humanos que "proporcionan insumos que permiten el desarrollo de las actividades primarias" (p. 144). Lo anterior fundamentado en la práctica de la administración que debe conducir al logro de determinados objetivos, por medio de las funciones de planeación, organización, dirección y control (Robbins y De Cenzo, 2002), que son generalmente aceptadas como las que componen el proceso administrativo.

En consecuencia, se prevé que, con el pensamiento estratégico, la dirección estratégica y la gerencia basada en el valor se logran resultados exitosos. Escenario que se espera obtener con la ejecución del programa de fortalecimiento que aquí se presenta.

\section{Metodología}

Para la caracterización de las empresas se desarrolló un estudio mixto, que exigió la ejecución de procedimientos previamente definidos (Hernández, Fernández y Baptista, 2010), de modo que se facilitara la recolección y el análisis simultáneo de datos cuantitativos y cualitativos. Se utilizó el diseño de triangulación concurrente (DITRIAC) que mencionan Hernández, Fernández y Baptista (2010), con un enfoque deductivo porque se realizó exploración y descripción de las empresas estudiadas a partir de postulados teóricos del pensamiento estratégico, la dirección estratégica y la gerencia basada en el valor.

El estudio también es exploratorio, descriptivo y aplicado. Exploratorio porque se examinó "un tema o problema de investigación poco estudiado, del cual 
se tienen muchas dudas o no se ha abordado antes" (Hernández, Fernández y Baptista, 2010, p. 127). Descriptivo porque se acudió a observación, entrevistas y cuestionarios estructurados para caracterizar las empresas curtidoras (Hernández, et al., 2010). Y aplicado porque se busca construir una propuesta que sea aprovechada por las empresas curtidoras.

\section{Variables}

Para la caracterización de las empresas se estudiaron las siguientes variables: a) oportunidades y amenazas: 1) económicas, 2) sociales, 3) culturales, 4) demográficas, 5) ambientales, 6) políticas, 7) gubernamentales, 8) legales, 9) tecnológicas y 10) competitivas; b) fortalezas y debilidades en: 1) contabilidad y finanzas; 2) procesos internos; 3) prácticas productivas; 4) logística; 5) prácticas comerciales; 6) uso de tecnologías limpias; 7) formación y crecimiento del talento humano y 8 ) proceso administrativo (planeación, organización, dirección y control).

\section{Participantes}

En atención a que "en ocasiones una misma investigación requiere una estrategia de muestreo mixta que mezcle varios tipos de muestra" (Hernández, et al., 2010 p. 443), se caracterizaron 13 empresas curtidoras agremiadas en la Asociación Ecológica de Chingacío - ASECHI del municipio de Chocontá. Esta cantidad se definió como muestra mixta no probabilística, a la que se aplicaron criterios de muestreo homogéneo, por oportunidad y por conveniencia. Por oportunidad y por conveniencia porque el representante legal ASECHI solicitó a la UNAD acompañamiento y asesoría para el fortalecimiento empresarial. Homogéneo porque las empresas desarrollan la misma actividad económica y tienen características similares. 


\section{Instrumentos}

En la caracterización de las empresas se utilizaron los siguientes instrumentos: a) acta de compromiso y confidencialidad para formalizar compromisos y garantizar la reserva sobre la información confidencial a la que se tuvo acceso; b) ficha de caracterización compuesta por varios formularios, mediante los cuales se recopiló información de las áreas funcionales de cada empresa, para lo que se acudió a preguntas cerradas con base en la escala de Likert y afirmaciones de opción múltiple con única respuesta (Hernández, et al., 2010); c) matrices EFI (de evaluación del factor interno) y EFE (de evaluación del factor externo) para clasificar oportunidades, amenazas, fortalezas y debilidades y d) matriz de registro de observaciones para describir otras características de las empresas que no se incluyen en los demás formatos como prácticas administrativas, conducta y actitudes de los empresarios y directivos.

Para el diseño del Programa de Acompañamiento para el Fortalecimiento a la Gestión Empresarial de Mipymes Curtidoras del Municipio de Chocontá (Cundinamarca) aún no se han elaborado instrumentos. Dicha labor se adelantará en la etapa "Estructuración de instrumento de diagnóstico", correspondiente a la fase de “instrumentación", cuyo objeto es diseñar un instrumento digital para el diagnóstico (tabla 2).

\section{Procedimientos}

En el proceso de caracterización de las empresas se recolectaron y analizaron datos cuantitativos y cualitativos de forma concurrente. En particular para: a) sensibilizar a los empresarios para describir el proceso; b) formalizar la participación con la suscripción del acta de compromiso y confidencialidad; c) visitar las plantas de producción; d) aplicar la ficha de caracterización; e) observar otras características de las empresas, las prácticas administrativas y "comportamientos concretos" para descubrir y comprobar"la asociación entre variables de investigación" (Méndez, 2006, p. 231); y f) procesar la información en la hoja de cálculo Excel. 
Para el diseño del Programa de Acompañamiento para el Fortalecimiento a la Gestión Empresarial de Mipymes Curtidoras del Municipio de Chocontá (Cundinamarca) se consultaron varios de los programas de fortalecimiento empresarial existentes en Colombia. Entre muchos que ofrecen diferentes organizaciones públicas se pueden mencionar el "Programa de Fortalecimiento Empresarial a las Micro y Pequeñas Empresas" del SENA; el "Programa de Transformación Productiva PTP" y el"Programa de Fortalecimiento Empresarial a las MIPYMES" del Ministerio de Comercio, Industria y Turismo - MCIT y el "Programa de Fortalecimiento Empresarial" del Instituto Distrital de Turismo de Bogotá. También se pueden mencionar algunos de los que ofrecen organizaciones privadas como el "Programa de Fortalecimiento Empresarial de la Cámara de Comercio de Bogotá", que ofrece la posibilidad de hacer un auto diagnóstico en línea; el programa de "Fortalecimiento Empresarial - Cafam"; el de la Fundación Amanecer; el de"Desarrollo empresarial" del Centro Alaya, "iniciativa de las Universidades Icesi y Valle, el Ministerio de Comercio, Industria y Turismo, la Fundación de Apoyo de la Universidad del Valle y Compañeros de las Américas". Igualmente, el programa "Fortalecimiento empresarial y regional" de Propaís, que es una entidad de carácter mixto.

\section{Método de análisis de datos}

Los datos recolectados para la caracterización de las empresas curtidoras fueron tabulados y sistematizados en una hoja de cálculo de Excel. Esta información sirvió para identificar fortalezas, oportunidades, debilidades y amenazas de cada empresa. Luego, con base en estadística descriptiva, se analizan los resultados y se establecen similitudes. Todo ello con base en el Diseño de Triangulación Concurrente DITRIAC, apropiado para "efectuar validación cruzada entre datos cuantitativos y cualitativos, así como [para] aprovechar las ventajas de cada método y minimizar sus debilidades" (Hernández, 2010, p. 612). A partir de la caracterización se elaboró la propuesta de acompañamiento para el fortalecimiento a la gestión que a continuación se describe. 


\section{Resultados}

Como se comentó en el planteamiento del problema, el primer objetivo específico de la investigación, diagnosticar a las empresas, ya se logró al realizar la caracterización de las empresas (tabla 1). A continuación se presentan los resultados correspondientes al segundo objetivo específico que consiste en diseñar el programa de acompañamiento para el fortalecimiento. Con base en la caracterización realizada, el Programa de Acompañamiento a la Gestión Empresarial de Mipymes Curtidoras del Municipio deChocontá(Cundinamarca) es el resultado de esta segunda etapa del proyecto de investigación.

\section{Programa de Acompañamiento para el Fortalecimiento a la Gestión Empresarial de Mipymes Curtidoras del Municipio de Chocontá (Cundinamarca)}

Para el desarrollo del programa es necesario tener en cuenta que en el proceso de producción todas las empresas curtidoras de Chocontá realizan los mismos procedimientos, que son: a) recepción, b) sacudir la sal, c) pesaje, d) remojo, e) sulfuración, f) descarnado, g) división de la carnaza y el pelo-flor, h) desencalado (quitar la cal), i) curtido, j) rebaje de calibre, k) teñido, I) secado, m) tolde (cubrimiento) y n) empaquetado. También utilizan los mismos materiales: sulfuro, cal, desencalantes, cromo, tintas, grasa y agua. Vale la pena resaltar que la estructura del programa está en función del estado de las empresas, razón por la cual se definen cuatro componentes generales: objetivo, participantes, metodología y fases.

Objetivo. Proporcionar acompañamiento para el fortalecimiento de la gestión empresarial y el mejoramiento de las prácticas productivas, logísticas y de comercialización. Con esto se busca promover el uso de tecnologías limpias, propiciar el acceso a mercados nacionales e internacionales y mejorar la competitividad de las Mipymes curtidoras de cuero del municipio de Chocontá. 
Lo anterior, a través de acciones concretas en el proceso administrativo (planeación, organización, dirección y control) y en las áreas funcionales (producción, mercadeo y ventas, contabilidad y finanzas, gestión del talento humano y administración). Como consecuencia del logro del objetivo se espera el mejoramiento de las condiciones laborales, económicas y sociales de empresarios, empleados y familiares de quienes se dedican al curtido de cueros.

Participantes. Para la ejecución del programa se definen diferentes actores y roles. Los actores serán empresarios, docentes y estudiantes de la Universidad Nacional Abierta y a Distancia- UNAD y la Decana de la Escuela de Ciencias Administrativas, Contables, Económicas y de Negocios - ECACEN de la UNAD. Los empresarios desempeñarán el rol de representantes de las empresas beneficiarias. La Decana fungirá como supervisora del programa. El autor del presente trabajo será el Director del programa. Y los docentes y estudiantes, según su perfil, serán los asesores designados para el desarrollo de las fases y etapas que conforman el programa.

Metodología. Una vez realizadas la sensibilización y la inscripción, se iniciará la ejecución de las fases subsiguientes. Para ello, de común acuerdo con el empresario, el asesor designado elaborará un cronograma de trabajo, visitas de asesoría y acompañamiento. Para la elaboración del diagnóstico se utilizarán el instrumento digital de diagnóstico que se diseñe. Dicho instrumento podrá ser similar a la ficha de caracterización utilizada en la etapa de diagnóstico a las 13 empresas curtidoras, la matriz de evaluación del factor externo - EFE, la matriz de evaluación del factor interno - EFI y la matriz de registro de observaciones utilizada en la caracterización. De todos modos, tal instrumento deberá ser digital y permitir la realización de diagnósticos de áreas funcionales y variables externas que afectan las empresas, facilitar el procesamiento de la información y generar informes de diagnóstico. Los modelos de informe de diagnóstico, de avance y de cierre serán diseñados por el director del programa; lo mismo ocurre con el acta de cierre del programa. 
Fases. El programa de Acompañamiento a la Gestión de las Mypimes curtidoras de Chocontá está compuesto por seis fases que, en términos generales, se convierten en su columna vertebral. Tales fases se describen en la tabla 2, en la que también se registran las etapas de cada fase y el tiempo estimado de duración.

Tabla 2. Fases y etapas del Programa de Acompañamiento a la Gestión Empresarial de Mipymes Curtidoras de Chocontá (Cundinamarca)

\begin{tabular}{|c|c|c|c|}
\hline 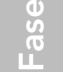 & Etapas & Descripción & Duración \\
\hline \multirow{3}{*}{ 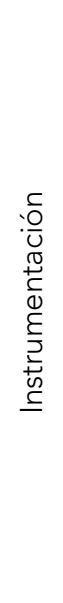 } & $\begin{array}{l}\text { Estructuración } \\
\text { instrumento de } \\
\text { diagnóstico }\end{array}$ & $\begin{array}{l}\text { Diseño de un instrumento digital para } \\
\text { realización de diagnóstico a todas las áreas } \\
\text { de la empresa y funciones administrativas. }\end{array}$ & $\begin{array}{l}\text { Dos (2) } \\
\text { meses }\end{array}$ \\
\hline & $\begin{array}{l}\text { Modelación plan } \\
\text { estratégico }\end{array}$ & $\begin{array}{l}\text { Construcción del modelo de plan estratégico } \\
\text { (formulación estrategias FO, FA, DO y DA), } \\
\text { calificación de objetivos estratégicos y de } \\
\text { estrategias y formulación de metas. }\end{array}$ & \\
\hline & $\begin{array}{l}\text { Modelación planes } \\
\text { tácticos }\end{array}$ & $\begin{array}{l}\text { Construcción del modelo de planes } \\
\text { tácticos (financiero o de inversión; de } \\
\text { negocios o de crecimiento y expansión; } \\
\text { de internacionalización; de marketing; de } \\
\text { formación (capacitación) y otros según, se } \\
\text { requiera). Se deben establecer actividades, } \\
\text { indicadores, plazos, recursos y responsables. }\end{array}$ & \\
\hline \multirow{3}{*}{ 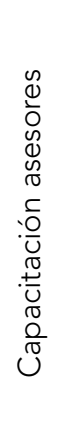 } & Definición Perfiles & $\begin{array}{l}\text { El director del programa estructura los } \\
\text { perfiles requeridos para los asesores que } \\
\text { ejecutarán el programa. }\end{array}$ & Un (1) mes \\
\hline & Selección Asesores & $\begin{array}{l}\text { Una vez definidos los perfiles, se convoca } \\
\text { estudiantes (pasantes) y docentes para } \\
\text { seleccionar los asesores. }\end{array}$ & $\begin{array}{l}\text { Dos (2) } \\
\text { meses }\end{array}$ \\
\hline & Capacitación & $\begin{array}{l}\text { Se brinda orientación al respecto de } \\
\text { objetivos, metodología, fases, formatos, } \\
\text { instrumentos, modelos procedimientos y } \\
\text { demás detalles del programa. }\end{array}$ & $\begin{array}{l}\text { Dos (2) } \\
\text { meses }\end{array}$ \\
\hline
\end{tabular}

continúa 


\begin{tabular}{|c|c|c|c|}
\hline \multirow{4}{*}{$\begin{array}{l}\frac{\pi}{0} \\
\frac{0}{0} \\
\frac{\pi}{0} \\
\frac{0}{0} \\
\frac{0}{2}\end{array}$} & Sensibilización & $\begin{array}{l}\text { Presentación del programa a los beneficiarios } \\
\text { por parte del director del programa. }\end{array}$ & $\begin{array}{l}\text { Una (1) } \\
\text { semana }\end{array}$ \\
\hline & Inscripción & $\begin{array}{l}\text { El empresario y el asesor designado } \\
\text { suscriben acta de inscripción, compromiso y } \\
\text { confidencialidad. }\end{array}$ & $\begin{array}{l}\text { Una (1) } \\
\text { semana }\end{array}$ \\
\hline & Diagnóstico & $\begin{array}{l}\text { El asesor recopila información para } \\
\text { establecer el estado en que se encuentra la } \\
\text { empresa }\end{array}$ & $\begin{array}{l}\text { Una (1) } \\
\text { semana }\end{array}$ \\
\hline & Informe de Diagnóstico & $\begin{array}{l}\text { El asesor procesa la información recopilada } \\
\text { y presenta informe al empresario para su } \\
\text { validación. }\end{array}$ & $\begin{array}{l}\text { Dos (2) } \\
\text { semanas }\end{array}$ \\
\hline \multirow{3}{*}{ 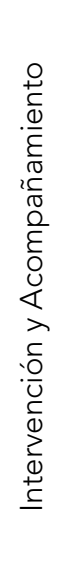 } & Planeación & $\begin{array}{l}\text { Con base en el informe de diagnóstico, en } \\
\text { forma conjunta, el asesor y el empresario } \\
\text { formulan un plan estratégico y los planes } \\
\text { tácticos requeridos. }\end{array}$ & Un(1) Mes \\
\hline & & $\begin{array}{l}\text { El empresario implementa los planes } \\
\text { formulados. }\end{array}$ & \\
\hline & $\begin{array}{l}\text { Implementación y } \\
\text { seguimiento }\end{array}$ & $\begin{array}{l}\text { El asesor designado acompaña y orienta } \\
\text { a empresario y funcionarios a cargo, } \\
\text { realiza seguimiento a la implementación } \\
\text { de los planes y acciones de mejoramiento } \\
\text { ejecutadas y presenta informes mensuales } \\
\text { a la dirección del programa. El informe } \\
\text { debe dar cuenta de los logros de las metas } \\
\text { propuestas. }\end{array}$ & Un (1) año \\
\hline \multirow{2}{*}{ 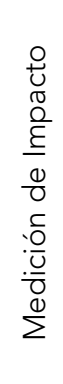 } & Revisión de Avances & $\begin{array}{l}\text { El asesor designado construye indicadores } \\
\text { para comparar el avance de la empresa } \\
\text { frente a los objetivos del programa. }\end{array}$ & Un (1) año \\
\hline & Informe de Avance & $\begin{array}{l}\text { El asesor presenta informe de fortalecimiento } \\
\text { a la gestión de la empresa, tanto al } \\
\text { empresario como al director del programa. } \\
\text { En caso de que la empresa no alcance } \\
\text { determinado nivel, debe recomendar las } \\
\text { acciones de mejoramiento que se requieran. }\end{array}$ & Un (1) mes \\
\hline \multirow{2}{*}{$\frac{\mathscr{Q}}{\frac{U}{U}}$} & Finalización del programa & $\begin{array}{l}\text { El empresario, el director del programa y el } \\
\text { asesor suscriben acta de finalización de la } \\
\text { participación de la empresa en el programa. }\end{array}$ & Un (1) mes \\
\hline & Informe de cierre & $\begin{array}{l}\text { El asesor presenta logros alcanzados, } \\
\text { limitaciones, lecciones aprendidas y } \\
\text { sugerencias para que la empresa continúe el } \\
\text { proceso de mejoramiento. }\end{array}$ & $\begin{array}{l}\text { Dos (1) } \\
\text { mes }\end{array}$ \\
\hline
\end{tabular}

Fuente: elaboración propia 


\section{Conclusiones}

Por sus aportes al PIB y la generación de empleo, la cadena productiva del cuero es un sector productivo muy importante para la economía colombiana. Sin embargo, en la actualidad enfrenta unas perspectivas desfavorables.

Para superar los desafíos que enfrentan las empresas curtidoras de cuero es necesario emprender programas de fortalecimiento a la gestión empresarial. Programas que deben fundamentarse en un pensamiento estratégico con enfoque sistémico, en el que los empresarios desarrollen "capacidad de síntesis, inteligencia intuitiva y creatividad con el fin de visualizar un futuro y articular la forma de llegar a él" (Román, 2010, p. 26).

Para la obtención de resultados exitosos en la ejecución del programa de fortalecimiento que propone esta investigación, es indispensable que los empresarios de las Mipymes curtidoras de cuero de Chocontá aporten los insumos que refiere Robert (2006), es decir: conocimientos, experiencia, opiniones, información subjetiva y los juicios objetivos de propietarios y administradores.

En ese orden de ideas, el programa de fortalecimiento a la gestión empresarial se fundamenta en aspectos del pensamiento estratégico, la dirección estratégica y la gestión basada en el valor; además permite que los curtidores de Chocontá entiendan la realidad del sector del cuero, formulen y ejecuten estrategias óptimas para el mejoramiento de sus empresas, de tal forma que estas generen valor no solo para ellos sino para todos los grupos de interés (Ortiz, 2003).

Antes de iniciar la ejecución del programa de fortalecimiento a la gestión empresarial es necesario estructurar un instrumento de diagnóstico, modelar un plan estratégico y unos planes tácticos, capacitar a los asesores que desarrollarán la intervención y el acompañamiento y preparar a los empresarios y empleados. Atendiendo a Dess y Lumpkin (2003), para la creación de ventajas competitivas, los curtidores de Chocontá deben analizar las decisiones y las acciones a emprender. 


\section{Recomendaciones}

El programa de fortalecimiento de la gestión empresarial de Mipymes curtidoras debe asumirse como proyección social y como acción que contribuya a la materialización del décimo postulado del decálogo de valores, definido en el Proyecto Académico Pedagógico Solidario - PAPS de la UNAD, según el cual la Universidad cree "en la importancia de trabajar para que la proyección social y la investigación generen mejores condiciones de vida a las poblaciones vulnerables del país" (UNAD, 2011, p. 35).

Para propiciar la implementación del programa que se propone es indispensable hacer seguimiento a lo ordenado en la sentencia No. 25000-23-27-0002001-90479-01(AP) del 28 de marzo de 2014 del Consejo de Estado (p. 1566), mediante la que se ordenó "la construcción y puesta en funcionamiento del Parque Ecoeficiente Industria de las curtiembres", proyectada en la vereda Chingacío de Chocontá, lugar en el que, se supone, se ubicarían los curtidores de cuero de Chocontá y Villapinzón.

Durante la ejecución del programa de fortalecimiento a la gestión empresarial de Mipymes curtidoras se deben recopilar y documentar las lecciones aprendidas, de manera que se mejoren los aspectos a que haya lugar para ofrecerlo a los empresarios del cuero del municipio de Villapinzón y posteriormente a otras áreas y sectores económicos del país.

\section{Referencias}

ACICAM. (2017). ¿Cómo va el sector? Enero a abril de 2017 [en línea]. Disponible en http://acicam.org/como-va-el-sector?download=259\%3Aabril-2017\&start=20

Caja de Compensación Familiar Cafam. (2017). Fortalecimiento Empresarial - Cafam [en línea]. Disponible en http://www.cafam.com.co/Empleabilidad/FortalecimientoEmpresarial 
Cámara de Comercio de Bogotá. (2017). Fortalecimiento empresarial [en línea]. Disponible en http://www.ccb.org.co/Preguntas-frecuentes/FortalecimientoEmpresarial/Fortalecimiento-Empresarial

Centro Alaya. (2017). Desarrollo empresarial [en línea]. Disponible en http://www.icesi. edu.co/cdee/centro-alaya/programas-de-fortalecimiento-empresarial.php

Consejo de Estado. (2014). Sección primera. Sentencia 2018698 del 28 de marzo de 2014. Disponible en http://190.24.134.114:8080/WebRelatoria/ce/index.xhtml

Chiavenato, I., \& Sapiro, A. (2011). Planeación estratégica: Fundamentos y aplicaciones. México: Mc Graw Hill.

David, F. (2013). Conceptos de administración estratégica. (Décimocuarta edición). México: Pearson Prentice Hall.

Flores A., Salazar, G. y Tapia, D. (2011). Administración Estratégica. Disponible en http://www.uaeh.edu.mx/docencia/P_Presentaciones/tlahuelilpan/administracion/ admin_estrategica/admin_estra.pdf

Fundación Amanecer. (2017). Fortalecimiento empresarial [en línea]. Disponible en http://www.amanecer.org.co/index.php/desarrollo-empresarial-y-productivo/ fortalecimiento-empresarial

Gutiérrez, J. (2008). Modelos Financieros con Excel. Herramientas para mejorar la toma de decisiones empresariales. Bogotá: ECOE EDICIONES. Segunda Edición. Disponible en http://fullseguridad.net/wp-content/uploads/2016/11/Modelosfinancieros-con-Excel-2da-Edic\%C3\%B3n-Jairo-Guti\%C3\%A9rrez-Carmona.pdf

Harrison, J. S., \& H. St John, C. (2002). Fundamentos de la Dirección Estratégica. Madrid: Ediciones Paraninfo S.A.

Hernández, R., Fernández, C. y Baptista, P. (2010). Metodología de la investigación. (Quinta edición). México, D.F.: McGraw-Hill. 
Hill \& McShane. (2008). Principles of Management [en línea]. Disponible en http:// instructors.coursesmart.com/0077222466/ii?readertab=search\&readersearch $=$ Principles+of+Management\&readerquery=(((Principles+of+Management+AND+(BOOKSHELF+OR+ARCHIVES)))+AND+ONLINESUBSCRIBED+)+AND+ISBN\%3d0077222466\#X2ludGVybmFsX0J2ZGVwRmxhc2hSZWFkZXI/ eG1saWQ9MDA3NzlyMjQ2Ni80

Instituto Distrital de Turismo. (2017). Programa de fortalecimiento empresarial [en línea]. Disponible en http://www.bogotaturismo.gov.co/eventos/programa-defortalecimiento-empresarial

Laudon, K. y Laudon, J. (2004). Sistemas de información gerencial [en línea]. Disponible en http://books.google.com.co/books?id=KD8ZZ66PF-gC\&pg=PA66\&dq=apoyo+d e+los+sistemas+de+informaci\%C3\%B3n+a+las+\%C3\%A1 reas+de+la+empresa\&hl= es\&sa=X\&ei=te-LU-rOM6rKsATtzIGYBA\&redir_esc=y\#v=onepage\&q=funciones\%20 de\%20la\%20empresa\&f=false

Méndez, C. (2006). Metodología, diseño y desarrollo del proceso de investigación con énfasis en ciencias empresariales. (Cuarta edición). Bogotá, D.C.: Limusa.

Minstzberg, H. Quinn, J. y Voyer, J. (1997). El proceso estratégico. Conceptos, contexto y casos [en línea]. (Primera edición). México: Pearson Prentice Hall. Disponible en: https://books.google.com.mx/books?hl=es\&lr=\&id=YephqTRD71IC\&oi=fnd\&pg=PR13\&dq=el+pensamiento+estrat\%C3\%A9gico+es+el+arte+de+ordenar+los+conocimientos+y+los+recursos+para+superar+esa+diferencia+tradicional+que+existe+entre+el+plan+y+el+resultado\&ots=FbGIJ31Xot\&sig=oiRgPkgJCXW_1P79obcxaT8WeVA\#v=onepage\&q=pensamiento\%20 estrat\%C3\%A9gico\&f=false

Ministerio de Comercio, Industria y Turismo (MCIT). (2017). Programa de fortalecimiento empresarial a las Mipymes [en línea]. Disponible en https://www.capitalcolombia. com/articulo/fortalecimiento_Mipymes. 
Ministerio de Comercio, Industria y Turismo (MCIT). (2017). Programa de transformación productiva [en línea]. Disponible en https://www.ptp.com.co/contenido/contenido. aspx?cat ID=607\&conID=1

Ortiz, H. (2003). Análisis financiero aplicado con análisis de valor agregado. Bogotá, D.C.: Universidad Externado de Colombia.

Porter, M. (1998). The competitive advantaje of nations. Whit a new introduction [en línea]. USA: Simon \& Schuster. Disponible en https://books.google.com.co/books?i-

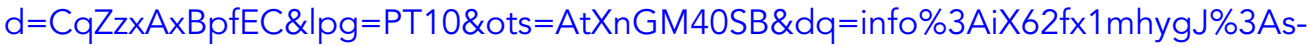
cholar.google.com\&lr\&pg=PT7\#v=onepage\&q\&f=false

Propaís. (2017). Fortalecimiento empresarial y regional de Propaís [en línea]. Disponible en http://propais.org.co/servicios-y-programas-de-propais/fortalecimiento-empresarial-y-regional/

Robbins, S. y De Cenzo, D. (2002). Fundamentos de administración: conceptos esenciales y aplicaciones [en línea]. Disponible en https://books.google.com.co/ books?id=yly3Ak0GLykC\&pg=PA6\&dq=planear, + organizar, + dirigir $+y+$ controlar\&hl=es\&sa=X\&ei=kncAVYm-CcHZggSO4IPgBQ\&redir_esc=y\#v=onepage\&q=planear\%2C\%20organizar\%2C\%20dirigir\%20y\%20controlar\&f=false

Robert, M. (2006). El nuevo pensamiento estratégico puro y libre [en línea]. México, D.F.: McGraw-Hill Interamericana. Disponible en http://bibliotecavirtual.unad.edu. co:2077/lib/unadsp/reader.action?ppg=1\&doclD=10433855\&tm=1498591583003

Romero, R., Noriega, S., Escobar, C. y Ávila, V. (2009). Factores críticos de éxito: una estrategia de competitividad. En Revista Cultura Científica y Tecnológica, año 6, núm. 31. Disponible en http://openjournal.uacj.mx/ojs/index.php/culcyt/article/ view/340/322

Universidad Nacional Abierta y a Distancia (UNAD). (2011). Proyecto académico pedagógico solidario. Versión 3.0. Disponible en http://thumano.unad.edu.co/portal/documentos/induccion/PAP\%20solidario\%20v3.pdf 
Román, O. (2010). El pensamiento estratégico. Una integración de los sentidos con la razón. En Revista Científica Guillermo de Ockham, 8(2). Disponible en revistas.usb. edu.co/index.php/GuillermoOckham/article/download/562/363

Román, O. (2011). La estrategia como un proceso mental. En Revista Gestión \& Desarrollo, 8(1). pp. 17-50. Disponible en http://revistas.usb.edu.co/index.php/GD/ article/view/1821/1577 
